\title{
Review Article \\ New Antihyperglycemic Drugs and Heart Failure: Synopsis of Basic and Clinical Data
}

\author{
Dirk von Lewinski, ${ }^{1}$ Ewald Kolesnik, ${ }^{1}$ Markus Wallner, ${ }^{1,2}$ \\ Michael Resl, ${ }^{3,4}$ and Harald Sourij ${ }^{5}$ \\ ${ }^{1}$ Department of Cardiology, Medical University of Graz, Auenbruggerplatz 15, 8036 Graz, Austria \\ ${ }^{2}$ Cardiovascular Research Center, Lewis Katz School of Medicine, Temple University, Philadelphia, PA 19140, USA \\ ${ }^{3}$ Department of Endocrinology, Medical University of Vienna, Währinger Gürtel 18-20, 1090 Vienna, Austria \\ ${ }^{4}$ Department of Internal Medicine, Hospital Barmherzige Brüder Linz, Seilerstätte 2, 4021 Linz, Austria \\ ${ }^{5}$ Department of Endocrinology and Diabetology, Medical University of Graz, Auenbruggerplatz 15, 8036 Graz, Austria
}

Correspondence should be addressed to Ewald Kolesnik; ewald.kolesnik@medunigraz.at

Received 14 April 2017; Accepted 12 July 2017; Published 15 August 2017

Academic Editor: Charbel Abi Khalil

Copyright (c) 2017 Dirk von Lewinski et al. This is an open access article distributed under the Creative Commons Attribution License, which permits unrestricted use, distribution, and reproduction in any medium, provided the original work is properly cited.

\begin{abstract}
The assessment of the cardiovascular safety profile of any newly developed antihyperglycemic drug is mandatory before registration, as a meta-analysis raised alarm describing a significant increase in myocardial infarction with the thiazolidinedione rosiglitazone. The first results from completed cardiovascular outcome trials are already available: TECOS, SAVOR-TIMI, and EXAMINE investigated dipeptidyl peptidase 4 (DPP-4) inhibitors, ELIXA, LEADER, and SUSTAIN-6 investigated glucagon-like peptide 1 (GLP-1) receptor agonists, and EMPA-REG OUTCOME and CANVAS investigated sodium-dependent glucose transporter 2 (SGLT-2) inhibitors. LEADER, SUSTAIN-6, EMPA-REG OUTCOME, and CANVAS showed potential beneficial results, while the SAVOR-TIMI trial had an increased rate of hospitalization for heart failure. Meanwhile, the same drugs are investigated in preclinical experiments mainly using various animal models, which aim to find interactions and elucidate the underlying downstream mechanisms between the antihyperglycemic drugs and the cardiovascular system. Yet the direct link for observed effects, especially for DPP-4 and SGLT-2 inhibitors, is still unknown. Further inquiry into these mechanisms is crucial for the interpretation of the clinical trials' outcome and, vice versa, the clinical trials provide hints for an involvement of the cardiovascular system. The synopsis of preclinical and clinical data is essential for a detailed understanding of benefits and risks of new antihyperglycemic drugs.
\end{abstract}

\section{Introduction}

Throughout the last decade, demonstration of glucose lowering efficacy was the primary basis for the approval of antihyperglycemic drugs. However, increasing concerns about the cardiovascular safety profile of already approved glucose lowering drugs or drugs under consideration for approval have emerged. In 2007, Nissen and Wolski published their metaanalysis describing a relative $43 \%$ increase in myocardial infarction with the use of thiazolidinedione rosiglitazone [1]. The Food and Drug Administration (FDA) and the European Medicines Agency (EMA) responded by mandating the demonstration of the cardiovascular safety profile of novel antihyperglycemic drugs, requiring a cardiovascular outcome trial [2]. This novel regulation has changed the landscape for clinical trials in the field of diabetes significantly and since 2008 more than 160,000 patients have been enrolled in cardiovascular outcome trials (Figure 1) [3]. Augmenting data on potential cardiovascular side effects of antidiabetic drugs is very valuable since millions of people are treated over many years. In most of these patients, multiple cardiovascular risk factors are commonly present, so lowering the risk for macrovascular complications is one of the major tasks in current multifactorial diabetes management. Over the last years besides the classical primary ischemic endpoints, heart failure has emerged as an increasingly important endpoint in 


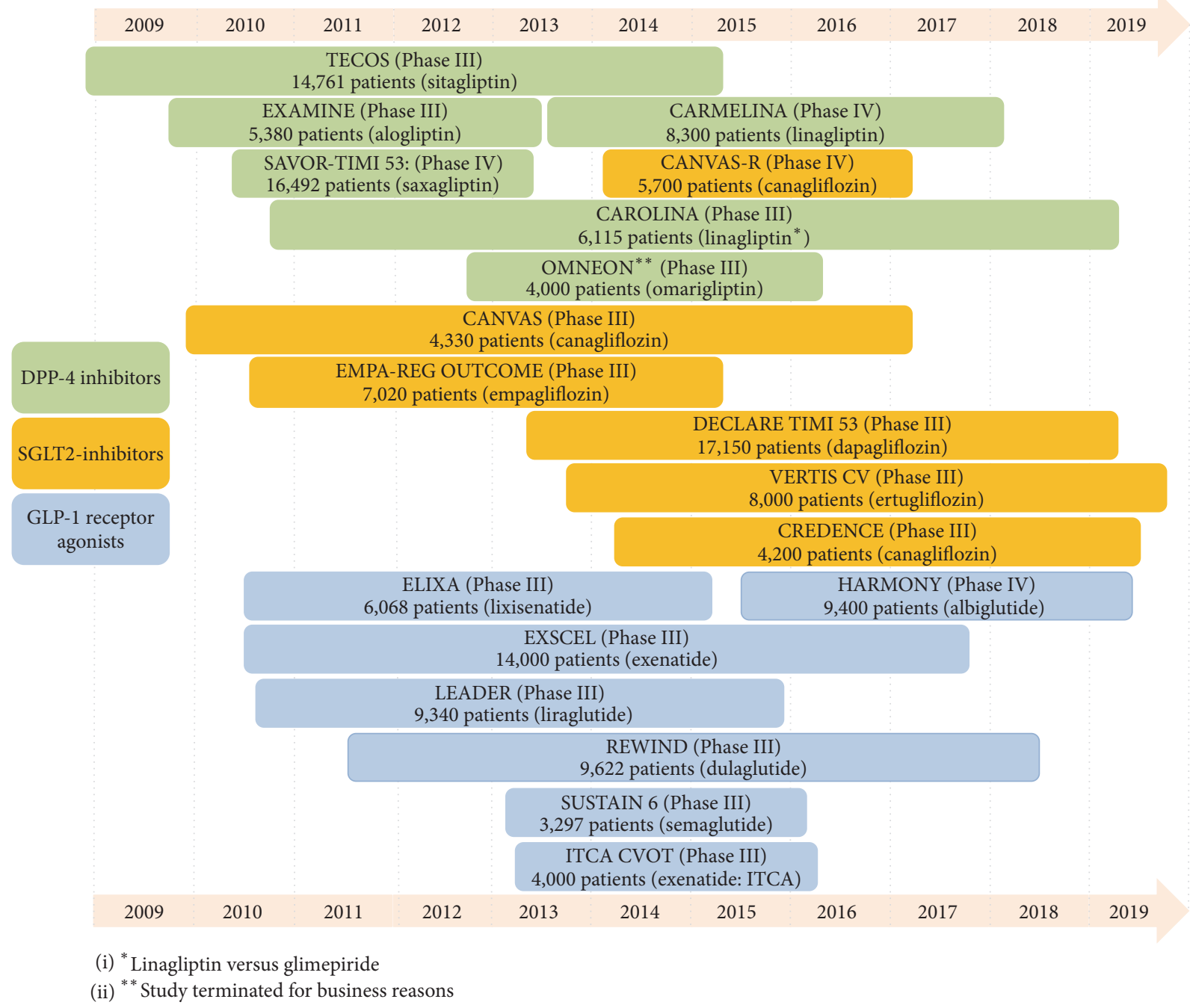

FIGURE 1: Timeline of already completed and still running cardiovascular safety trials. Green: DPP-4 inhibitors, orange: SGLT-2 inhibitors, and blue: GLP-1 receptor agonists. Name and number of planned included patients are given. All trials tested drugs versus placebo except the CAROLINA trail (linagliptin versus glimepiride).

diabetes outcome trials. Diabetes is a major risk factor for the development of heart failure [4], with approximately $22 \%$ of subjects with type 2 diabetes at an age above 65 years having a heart failure diagnosis [5].

Since 2013, eight of the FDA and EMA mandated trials have reported their results. There is no doubt that major cardiovascular events (MACE), death, and heart failure are indeed robust clinical endpoints; however, some of the results such as the potential heart failure signal for the dipeptidyl peptidase 4 (DPP-4) inhibitor saxagliptin in SAVORTIMI 53 or the pronounced cardiovascular benefit of the sodium-dependent glucose transporter 2 (SGLT-2) inhibitors empagliflozin and canagliflozin were rather surprising. Interestingly, there is little mechanistic insight to derive from these outcome trial data explaining cardiovascular harm or benefit. To sufficiently power these outcome trials while keeping the number of subjects and follow-up duration within acceptable limits, patients with diabetes and high cardiovascular risk or previously diagnosed atherosclerotic disease are randomized in these trials. However, the majority of patients with diabetes in routine care do not have a cardiovascular risk as high as represented by these trials [6].

This must be kept in mind, especially when findings from these outcome trials are extrapolated to patients with low cardiovascular risk. Performing outcome trials in the primary prevention setting would be important to inform future diabetes treatment, although this is a challenging task: given a MACE rate of approximately one-third as compared to subjects in the secondary prevention setting, trials in low cardiovascular risk patients would need to last longer, include more subjects, or combine both approaches, leading to a significant increase in the costs for such trials. Therefore, the synopsis of outcome data and results of basic research on cell and tissue level in models with elevated or not-elevated 
cardiovascular risk are of relevance and discussed in this review.

\section{Diabetic Heart}

Heart failure in diabetes represents a multifactorial problem resulting from a variety of cardiotoxic factors, such as coronary artery disease, hypertension, and direct harmful effects of glucose on the myocardium [7]. Besides well characterized macrovascular effects leading to coronary heart disease and corresponding clinical events, there is increasing data suggesting that there are direct associations between diabetes and heart failure. A 2-fold higher risk of heart failure in male diabetics and a 5 -fold increase in risk in female patients with diabetes have already been demonstrated in the Framingham study [8] and this association is of particular importance in younger patients [5]. The underlying mechanisms include but are not limited to increased interstitial and perivascular fibrosis. This histological pattern was considered the basis for the term "diabetic cardiomyopathy" in the early 1970s [9]. This type of fibrosis is independent of coronary artery disease or hypertension [10]. Nonetheless, diabetic cardiomyopathy remains only moderately understood. Advanced glycation end products (AGE) [11] and increased content crosslinking of collagen seem to play a significant role [12-14]. Besides histological findings, calcium homeostasis is probably affected directly as indicated by lower activity levels of the sarco/endoplasmic reticulum $\mathrm{Ca}^{2+}$-ATPase 2a (SERCA2a) in diabetic hearts [15]. Moreover, SERCA2a is a major regulator of glucose transport in the healthy and diabetic heart via calcium mediated glucose transporter (GLUT) type 4 translocation [16].

There is robust evidence that metabolic abnormalities underlie the impaired myocardial function in heart failure. Metabolic parameters such as the adenosine triphosphate to phosphocreatine ratio (ATP/PCr) have been shown to predict outcome even better than left ventricular ejection fraction (LV-EF) or the clinical NYHA class [17]. In addition, changes in myocardial metabolism show direct and acute effects on mechanical performance and this effect seems to be of particular importance in human myocardium. Insulin administration itself exerts positive inotropic effects in human ventricular myocardium via $\mathrm{Ca}^{2+}$-dependent and $\mathrm{Ca}^{2+}$-independent mechanisms. Both mechanisms raise the load of the sarcoplasmic reticulum (SR) resulting in an increase of systolic $\mathrm{Ca}^{2+}$-transients as well as an increase in myofilament sensitivity [18]. The metabolic changes upon insulin administration could be traced back to altered GLUT4 translocation and SGLT- 1 activation $[19,20]$. Additionally, insulin administration does not only result in acute functional effects, but also triggers various approaches modifying the energy substrate metabolism via an increased rate of pyruvate supply, as shown in vitro as well as in vivo [21,22].

Heart failure and diabetes interact bidirectionally. Besides an HbAlc dependent increased risk of developing heart failure in patients with diabetes mellitus, the prevalence of diabetes in heart failure patients is known to increase markedly over time (3.8\% per year) [23, 24]. Experimental data provides insight into substance-specific effects of glucose lowering therapy in heart failure. So far, with respect to the single classes of antidiabetic drugs and the related individual substances, the amount and quality of available experimental data are heterogeneous.

\section{DPP-4 Inhibitors}

While sitagliptin, alogliptin, and saxagliptin were shown to be safe for the cardiovascular system in terms of the MACE, cardiovascular death, and heart failure endpoints, the SAVOR-TIMI 53 trial showed a rather surprising signal for an increased risk for hospitalization of heart failure in the saxagliptin group, especially in the subgroups of impaired renal function and preexisting heart failure [25]. A similar trend could be observed for alogliptin in the EXAMINE trial (EXAMINE), albeit not statistically significant. In contrast, TECOS did not show an increased rate for heart failure hospitalizations after sitagliptin administration, suggesting a potential difference between members of the DPP- 4 inhibitor class. The cardiovascular outcome trials CARMELINA and CAROLINA (both for linagliptin) are still running and results are expected in 2018 and 2019, respectively. Recent metaanalyses including the finished major and many smaller cardiovascular safety studies for DPP-4 inhibitors have different conclusions, ranging from no increased risk for the hospitalization of heart failure after DPP4 inhibitor use [26] to an increased risk [27].

However, studies that examine the potential pleiotropic and nonglycemic effects of DPP-4 inhibitors on various cells and tissues may help to understand and interpret the difference in the observed cardiovascular side effects in some of the clinical trials. Recently, many reviews have tried to clarify the effects caused by DPP-4 inhibitors. They interact strongly with the heart, vascular system, kidney, liver, neuroendocrine system, immune system, and hematopoietic system affecting hormones or second messengers like brain natriuretic peptide (BNP), substance $\mathrm{P}$, activation of chemokine and cytokine pathways, intracellular calcium concentrations, and the release of nitric oxide (NO) shown in different animal models in vivo and ex vivo [28-32]. Interactions of DPP-4 inhibitors with the cardiovascular system and cardiomyocytes were successfully revealed, yet a direct link between DPP-4 inhibitors and its effects on cardiac contractility and/or electrophysiological function is still unknown, and the corresponding downstream mechanisms have yet to be determined. Therefore, studies that explored effects of DPP-4 inhibitors on cardiovascular system are of particular interest.

For saxagliptin, overwhelming potential beneficial effects are reported in literature: it reduces the damage of blood vessels via the amelioration of the availability of $\mathrm{NO}$ and the reduction of cyclooxygenase-1-action derived vasoconstriction caused by induced type-2 diabetes mellitus in mice [33] and, similarly, leads to a restoration of damaged mitochondrial vascular function in diabetic rats [34]. Additionally, a reduction of blood pressure by increasing the bioavailability of NO in spontaneous hypertensive rats [35] and an improvement of cardiac function after myocardial infarction independent of glucose lowering [36] could be demonstrated 
in diabetic rats. One study clarified that saxagliptin alters the cGMP-PKG-PDE5 axis in a swine model that mimicked heart failure with preserved ejection fraction (HFpEF) by aortic banding thus preventing left ventricular damage and improving left ventricular systolic and diastolic function [37]. Another study that explored the effects of saxagliptin on human multicellular myocardium and guinea pig ventricular cardiomyocytes revealed a negative inotropic potential, the prolongation of the action potential duration, and the occurrence of arrhythmias although the exact mechanism has not yet been determined [38].

Similar effects are reported for sitagliptin in diabetic rats. Sitagliptin improved endothelial function [39] and attenuated cardiac remodeling without affecting systolic function after myocardial infarction [40] while, in normoglycemic rats with induced myocardial infarction, sitagliptin prevented fatal arrhythmias by attenuating GIP-dependent resistin signaling [41] and in a PKA-dependent pathway [42]. Moreover, sitagliptin attenuated changes in the electrophysiological function in hypertensive rats [43] and counteracted induced HFpEF by improving the diastolic function, decreasing the generation of reactive oxygen species, and reducing proinflammatory biomarkers in the myocardium thus lowering mortality $[44,45]$. Similarly, one study proved the reduction of parameters of diastolic dysfunction and myocardial stiffness via the cGMP-PKG pathway after sitagliptin administration in obese diabetic mice [46].

Alogliptin could restore cardiac remodeling and prevent apoptosis via a cAMP-Epacl dependent and protein PKAindependent mechanism in a model of ventricular pressure overload [47] and inhibited inflammation in arteries that sustained damage by high LDL concentrations [48] in mice. The reported potential beneficial effects might also be present in humans; one trial with a small number of participants showed increased coronary flow reserve and improved left ventricular ejection fraction in patients with type- 2 diabetes and coronary artery disease within three months of alogliptin use [49].

For vildagliptin, conflicting results are reported: one study failed to show potential protective effects on cardiac function after myocardial infarction which thereby followed cardiac remodeling despite increased levels of active glucagon-like peptide 1 (GLP-1) in rats [50]. In contrast, other studies suggested that vildagliptin might reduce infarct size and preserve left ventricular ejection fraction by reducing reactive oxygen species in a rat model of ischemia/reperfusion [51] and preventing hypertrophy of the left ventricle after continuous infusion of isoproterenol in rats by the inhibition of inflammatory markers [52]. Additionally, vildagliptin exerts effects via $\mathrm{NO}$ and the endothelial NOsynthase (eNOS) leading to an improved vascularization in a mouse model with surgical induced ischemia [53]. Focusing on the cardiovascular system, vildagliptin seems to exert similar effects as sitagliptin [54]. However, no large cardiovascular outcome trial for vildagliptin is being performed.

Finally, linagliptin improves diastolic function in a model of HFpEF in obese rats via an elevated expression of eNOS and improved SERCA2a activity [55]. The effect on eNOS availability could be demonstrated in nonobese mice as well
[56]. Linagliptin also reduced angiotensin and glucose induced collagen formation in cardiac fibroblasts of mice by an anti-inflammatory mechanism (via NFkB) [57].

\section{GLP-1 Receptor Agonists}

The first cardiovascular outcome trial on glucagon-like peptide-1 receptor agonists was the ELIXA trial, which was designed to assess the effects of lixisenatide on the cardiovascular outcome in patients with type- 2 diabetes mellitus who had an acute coronary event within 180 days of screening. For the primary composite endpoint (cardiovascular death, myocardial infarction, and stroke), as well as for hospitalization for heart failure, no significant difference was observed between the treatment and placebo group [58]. The LEADER trial assessed the cardiovascular safety of liraglutide in patients with type- 2 diabetes mellitus and a HbAlc $\geq$ $7 \%$. Of the total enrolled subjects, $81.3 \%$ had preexisting cardiovascular diseases. Liraglutide significantly reduced the rate of the first occurrence of the primary endpoint (cardiovascular death, nonfatal myocardial infarction, or nonfatal stroke) and all-cause mortality. The rates of nonfatal stroke, myocardial infarction, and hospitalization for heart failure were nonsignificantly lower in the liraglutide group compared to the placebo group [59]. In the SUSTAIN-6 trial (semaglutide) patients with type-2 diabetes mellitus and established cardiovascular diseases, chronic heart failure, or chronic kidney disease, or $\geq 60$ years with at least one cardiovascular risk factor, were enrolled. Semaglutide significantly reduced the risk for the primary endpoint (first occurrence of cardiovascular death, nonfatal myocardial infarction, or nonfatal stroke). The protective effect of semaglutide on composite endpoints seems to be mainly driven by the reduction of nonfatal stroke [60]. The results of LEADER and SUSTAIN-6 continue to hold promise that GLP-1 receptor agonists might improve CV morbidity in patients with type2 diabetes mellitus. However, we do not yet fully understand the reasons for the diverging results in the currently published trials. Differences in the duration of action (short acting substances such as lixisenatide versus longer acting drugs like liraglutide or semaglutide) or differences within the amino acid sequences of the peptides are currently being discussed. Further insight will be gained from the imminent presentation of the EXSCEL trail [61].

GLP-1 is an incretin peptide hormone primarily synthesized by intestinal L cells [62]. It is released into the circulation in response to food intake, leading to glucosedependent insulin release and glucagon suppression. GLP$1(7-36) \mathrm{NH}_{2}$, with a half-life of 2 minutes, is the primary active isoform that is rapidly degraded by DPP-4 to GLP$1(9-36) \mathrm{NH}_{2}$ [63], a GLP-1 receptor antagonist [64]. Besides increased insulin secretion, GLP-1 receptor activation leads to an inhibition of gastric and small bowel motility, reduces appetite, and subsequently leads to weight loss [65]. In addition, human data suggests that this drug class improves cardiac function in patients with congestive heart failure, ameliorates endothelial dysfunction, and reduces the infarct size after ST-segment-elevation myocardial infarction [6669] 
The GLP-1 receptor is a seven transmembrane, G proteincoupled receptor (GPCR), and is positively coupled to adenylate cyclase through $\mathrm{G} \alpha_{\mathrm{s}}$-containing $\mathrm{G}$ proteins, which catalyze the conversion of ATP to cAMP. Increased cytosolic cAMP leads to activation of second messenger pathways including PKA, Epac2, and ERK-1/2 [70]. Beneficial effects of GLP-1 receptor agonists have been attributed to direct action on myocardium, with the majority of these effects reported in ventricular cardiomyocytes. However, there are conflicting reports regarding GLP-1 receptor expression in cardiac tissue. Recent studies in mice and rats revealed that the GLP1 receptor is exclusively localized in atrial cardiomyocytes [71-73]. Wallner et al. reported GLP-1 receptor expression in human right and left ventricular myocardium, although the expression levels were significantly lower compared to right atrial tissue [74]. This discrepancy between human and rodent tissue could be explained by species-related differences, such as those that exist for the SGLT, which is expressed in human myocardium but is undetectable in the myocardium of most species [20].

A recent study in normo- and hypertensive mice suggested that GLP-1 receptor activation in atrial cardiomyocytes increased cAMP levels, promoted Epac2 translocation to the membrane, and increased ANP secretion [71]. Epac2 functions in a PKA-independent manner and, therefore, represents a novel mechanism for governing signaling specificity within the cAMP cascade [75]. A recent study reported significant Epac2 translocation from the cytosol to the cell membrane after GLP-1 receptor activation in human atrial myocardium [74]. Epac2 activation increases phosphorylation of cardiac troponin $\mathrm{I}$ (cTnI) in a PKC-dependent manner resulting in increased myofilament $\mathrm{Ca}^{2+}$ sensitivity and contractility [76]. GLP-1 receptor agonists significantly increased developed force in human atrial trabeculae, whereas Exendin(9-39) $\mathrm{NH}_{2}$, a GLP-1 receptor antagonist, and $\mathrm{H}-89$, a PKA inhibitor, blunted the inotropic effect of exenatide. In addition, exenatide (a synthetic GLP-1 receptor agonist that is resistant to the degradation by DPP4) increased PKA-dependent phosphorylation of phospholamban (PLB) and GLUT-1 translocation, but not GLUT-4 translocation [74]. $\beta$-Arrestin signaling downstream of GLP1 receptor activation is another potential mechanism to increase cardiac contractility. $\beta$-arrestin, which is wellknown for contributing to the termination of GPCR signaling [77], might regulate cardiac function and increase cardiac contractility via $\beta$-arrestin-mediated processes [78-80]. Novel "biased ligands" that selectively recruit $\beta$-arrestin independent of $\mathrm{G}$ protein-mediated signaling have been described for the angiotensin II Type 1A receptor (AT1AR) [78] and the $\beta 1$-adrenergic receptor ( $\beta 1 \mathrm{AR}$ ) [80]. However, Wallner et al. showed that $\beta$-arrestin signaling downstream of GLP-1 receptor activation does not contribute to the positive inotropic effect in human atrial myocardium [74].

\section{SGLT-2 Inhibitors}

In 2015, the EMPA-REG-OUTCOME trial demonstrated a significant reduction in MACE and all-cause mortality in subjects treated with the SGLT-2 inhibitor empagliflozin
[81]. Moreover, this landmark trial showed a 35\% relative reduction in the rate of heart failure hospitalization in the empagliflozin group, an effect occurring very quickly after initiating treatment. These findings on MACE and heart failure hospitalization were confirmed in the recently published data from the CANVAS program with canagliflozin [82]. However, cardiovascular and all-cause mortality were not significantly reduced by canagliflozin, in contrast to empagliflozin. Currently, several hypotheses are being discussed for the findings in the SGLT-2 inhibitor trials. These include hemodynamic changes and increased hematocrit that are caused by a diuretic effect or changes in the cardiac fuel metabolism by an improved uptake of $\beta$-hydroxybutyrate under conditions of persistent hyperketonemia, all induced by SGLT-2 inhibitors. Particularly ischemic and therefore endangered myocardium may benefit from these effects [83, 84].

For SGLT-2 inhibitors, the most recent class of antidiabetic drugs established for clinical use, there is little data on cardiovascular side effects in animal models or in vitro settings available. This may be a consequence of the fact that the SGLT-2 receptor is not expressed in myocardial tissue [20, 81, 85]. Mechanistically, cardiovascular side effects of SGLT-2 inhibitors could occur either via unselective binding of compounds to SGLT-1, which is not the case for most of the members of this drug class, or via receptor independent effects. Interestingly, the pattern of intracellular mechanisms seems to be different for various class members.

Activation of AMPK, for example, has only been shown for canagliflozin but not for dapagliflozin or empagliflozin [86]. However, a pathway most likely influenced by all SGLT2 inhibitors in cardiomyocytes is the $\mathrm{Na}^{+} / \mathrm{H}^{+}$exchanger 1 (NHE1) mediated decrease in intracellular $\mathrm{Na}^{+}$and $\mathrm{Ca}^{2+}$, although this has only been reported for empagliflozin so far. Decreased intracellular $\mathrm{Ca}^{2+}$ is likely to result in a negative inotropic effect; however, this is not necessarily the case if both systolic and diastolic $\mathrm{Ca}^{2+}$ decrease and the $\mathrm{Ca}^{2+}$ transient remains stable. Moreover, Baartscheer et al. did show that mitochondrial $\mathrm{Ca}^{2+}\left(\left[\mathrm{Ca}^{2+}\right]_{\mathrm{m}}\right)$ did significantly increase upon empagliflozin administration. $\left[\mathrm{Ca}^{2+}\right]_{\mathrm{m}}$ signaling is critical for energy production as well as the activation of cell death pathways which are implicated in the development of heart failure [87]. These three changes in intracellular ion homeostasis counteract the alterations typically seen in heart failure models (e.g., elevated levels of intracellular $\mathrm{Na}^{+}$ and $\mathrm{Ca}^{2+}$ and reduced levels of mitochondrial $\mathrm{Ca}^{2+}$ in heart failure) and might thus explain at least in part beneficial effects as seen in the EMPA-REG-OUTCOME trial [81].

Elevated diastolic $\mathrm{Ca}^{2+}$ also results in impaired relaxation and therefore diastolic dysfunction. Interestingly, empagliflozin significantly improved diastolic function in a rodent model of diabetes and reduced the expression of profibrotic and prohypertrophic proteins [88]. These effects could not be explained by reduced blood pressure levels as reported in several other models after SGLT-2 treatment, indicating towards a direct myocardial effect.

This idea is also supported by the finding that dapagliflozin but not pioglitazone significantly improves cardiac 


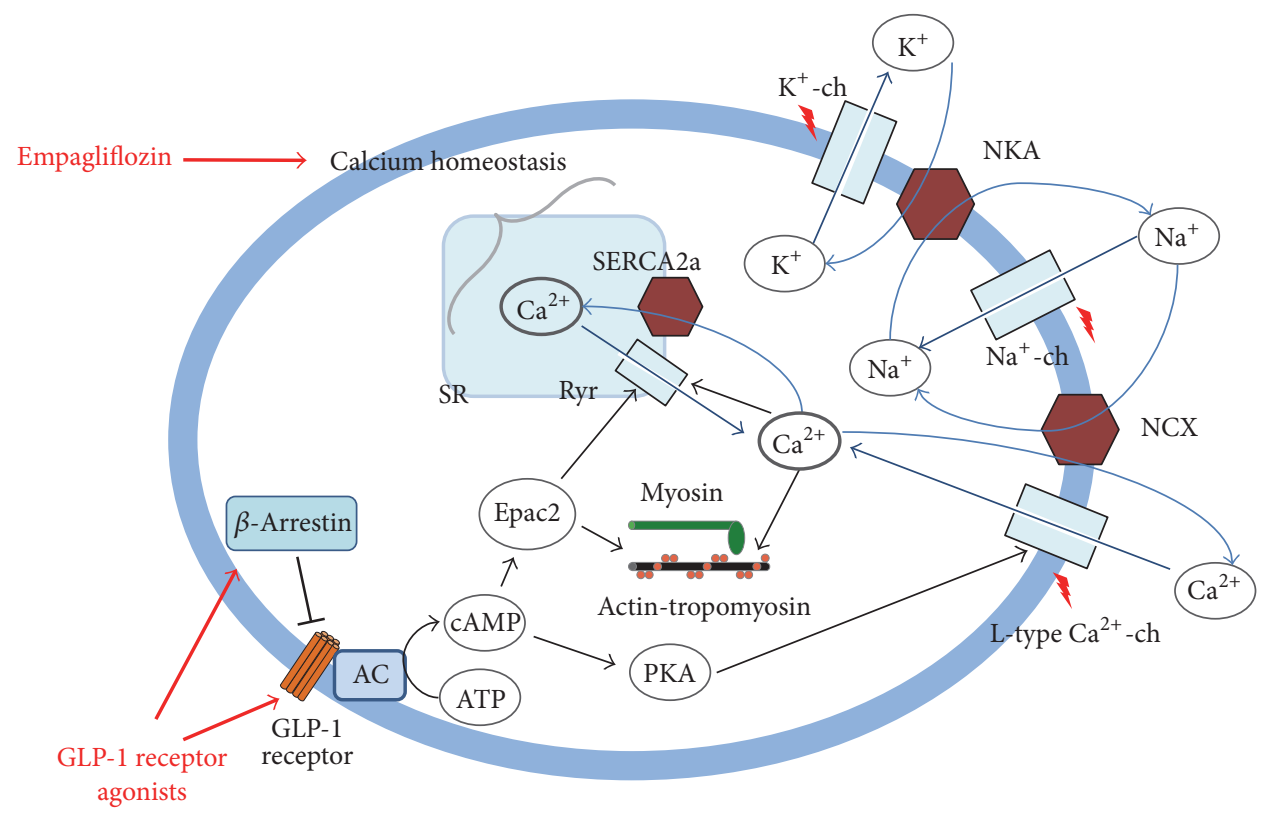

FIGURE 2: Interactions of antidiabetic drugs with cardiomyocytes: the well-established downstream mechanism of GLP-1 receptor agonists alters intracellular $\mathrm{Ca}^{2+}$ transients via a PKA-dependent activation of L-type $\mathrm{Ca}^{2+}$ channels and Epac2-dependent activation of the ryanodine receptor. For empagliflozin, potential downstream mechanisms are still unknown, yet there is strong evidence that the $\mathrm{Ca}^{2+}$ homeostasis is influenced. Possible downstream mechanisms of DPP-4 inhibitors are also still unknown. The wide interactions with cardiomyocytes via miscellaneous second messengers are not shown. $\left(\mathrm{Na}^{+}-\mathrm{ch}\right.$ : voltage gated sodium channel, $\mathrm{K}^{+}$-ch: voltage gated potassium channel, Ryr; ryanodine receptor, NCX: sodium-calcium exchange pump, and NKA: sodium-potassium exchange pump).

function in a mouse model despite comparable glucose lowering effects. Ejection fraction and isovolumetric relaxation time were not altered in pioglitazone, but E/A ratio and ventricular hypertrophy were both slightly improved.

\section{Metformin}

In the United Kingdom Prospective Diabetes Study (UKPDS), 342 patients with an ideal body weight greater than $120 \%$ were randomly assigned to an intensive treatment with metformin or conventional treatment. A 39\% relative risk reduction in fatal and nonfatal myocardial infarction $(p=0.010)$ and a $36 \%$ relative risk reduction in all-cause mortality ( $p=0.011$ ) were recorded in this study arm [89]. This finding in a limited number of patients is supported by data from a meta-analysis performed with randomized clinical trials data [90] suggesting a cardiovascular benefit associated with metformin. A larger trial investigating the effect of metformin in nondiabetic hyperglycemia is currently ongoing (ISRCTN 34875079).

\section{Conclusion}

With new and emerging primarily antihyperglycemic drugs, the intersection of antidiabetic treatment and cardiovascular therapy is progressing. Besides modulating diabetes as a cardiovascular risk factor several new antidiabetic drugs imply direct cardiovascular effects and in some cases these effects seem to directly affect myocardial tissue (Figure 2). Cardiovascular outcome trials requested by the FDA and
EMA were designed to test for global and rather indirect cardiovascular effects and the mechanistic basis for the beneficial findings in some of these trials remain to be elucidated. Although these trials are called placebo-controlled trials, subjects in the control arm receive usual diabetes care excluding compound/drug class used in the active arm and all these trials aim for glycemic equipoise in both groups in order to exclude cardiovascular effects which could be due to differences in glycemic control [91].

Global assessment of cardiovascular outcome in usually short- to mid-term trials in particular high risk populations, however, might miss distinct intracellular effects of new antidiabetic drugs that could mediate more specific, positive or negative, effects on excitation-contraction coupling, contractility, metabolism, or energetics resulting in altered structural or functional properties.

An in-depth examination of cardiovascular outcome data in conjunction with basic science data is critical for a detailed understanding of benefits and risks of new antihyperglycemic drugs.
Abbreviations
DPP-4: Dipeptidyl peptidase 4
EMA: European Medicines Agency
eNOS: Endothelial NO-synthase
FDA: Food and Drug Administration
GLP-1: Glucagon-like peptide 1
GLUT: Glucose transporter
HFpEF: Heart failure with preserved ejection fraction 
MACE: Major adverse cardiac event

NO: $\quad$ Nitric oxide

SERCA2a: Sarco-/endoplasmic reticulum $\mathrm{Ca}^{2+}$ ATPase

SGLT: Sodium-dependent glucose transporter.

\section{Conflicts of Interest}

The authors declare that there are no conflicts of interest regarding the publication of this paper.

\section{Acknowledgments}

The authors thank Deborah M. Eaton for careful linguistic revision of the manuscript.

\section{References}

[1] S. E. Nissen and K. Wolski, "Effect of rosiglitazone on the risk of myocardial infarction and death from cardiovascular causes," The New England Journal of Medicine, vol. 356, no. 24, pp. 24572471, 2007.

[2] M. A. Bethel and H. Sourij, "Impact of FDA guidance for developing diabetes drugs on trial design: From policy to practice," Current Cardiology Reports, vol. 14, no. 1, pp. 59-69, 2012.

[3] R. R. Holman, H. Sourij, and R. M. Califf, "Cardiovascular outcome trials of glucose-lowering drugs or strategies in type 2 diabetes," The Lancet, vol. 383, no. 9933, pp. 2008-2017, 2014.

[4] D. D. Schocken, E. J. Benjamin, G. C. Fonarow et al., "Prevention of heart failure: a scientific statement from the American Heart Association Councils on epidemiology and prevention, clinical cardiology, cardiovascular nursing, and high blood pressure research; Quality of Care and Outcomes Research Interdisciplinary Working Group; and Functional Genomics and Translational Biology Interdisciplinary Working Group," Circulation, vol. 117, no. 19, pp. 2544-2565, 2008.

[5] A. G. Bertoni, W. G. Hundley, M. W. Massing, D. E. Bonds, G. L. Burke, and D. C. Goff Jr., "Heart failure prevalence, incidence, and mortality in the elderly with diabetes," Diabetes Care, vol. 27, no. 3, pp. 699-703, 2004.

[6] K. Iglay, H. Hannachi, P. J. Howie et al., "Prevalence and coprevalence of comorbidities among patients with type 2 diabetes mellitus," Current Medical Research and Opinion, vol. 32, no. 7, pp. 1243-1252, 2016.

[7] D. S. H. Bell, "Heart failure: the frequent, forgotten, and often fatal complication of diabetes," Diabetes Care, vol. 26, no. 8, pp. 2433-2441, 2003.

[8] W. B. Kannel, M. Hjortland, and W. P. Castelli, "Role of diabetes in congestive heart failure: the Framingham study," The American Journal of Cardiology, vol. 34, no. 1, pp. 29-34, 1974.

[9] S. Rubler, J. Dlugash, Y. Z. Yuceoglu, T. Kumral, A. W. Branwood, and A. Grishman, "New type of cardiomyopathy associated with diabetic glomerulosclerosis," The American Journal of Cardiology, vol. 30, no. 6, pp. 595-602, 1972.

[10] T. J. Regan, M. M. Lyons, S. S. Ahmed et al., "Evidence for cardiomyopathy in familial diabetes mellitus," Journal of Clinical Investigation, vol. 60, no. 4, pp. 884-899, 1977.

[11] A. Goldin, J. A. Beckman, A. M. Schmidt, and M. A. Creager, "Advanced glycation end products: sparking the development of diabetic vascular injury," Circulation, vol. 114, no. 6, pp. 597$605,2006$.

[12] V. W. Fischer, H. B. Barner, and L. S. Larose, "Pathomorphologic aspects of muscular tissue in diabetes mellitus," Human Pathology, vol. 15, no. 12, pp. 1127-1136, 1984.

[13] K. H. Van Hoeven and S. M. Factor, "A comparison of the pathological spectrum of hypertensive, diabetic, and hypertensivediabetic heart disease," Circulation, vol. 82, no. 3, pp. 848-855, 1990.

[14] S.-I. Nunoda, A. Genda, N. Sugihara, A. Nakayama, S. Mizuno, and R. Takeda, "Quantitative approach to the histopathology of the biopsied right ventricular myocardium in patients with diabetes mellitus," Heart and Vessels, vol. 1, no. 1, pp. 43-47, 1985.

[15] D. D. Belke and W. H. Dillmann, "Altered cardiac calcium handling in diabetes," Current Hypertension Reports, vol. 6, no. 6, pp. 424-429, 2004.

[16] A. P. Waller, A. Kalyanasundaram, S. Hayes, M. Periasamy, and V. A. Lacombe, "Sarcoplasmic reticulum Ca2+ ATPase pump is a major regulator of glucose transport in the healthy and diabetic heart," Biochimica et Biophysica Acta - Molecular Basis of Disease, vol. 1852, no. 5, pp. 873-881, 2015.

[17] J. S. Ingwall and R. G. Weiss, "Is the failing heart energy starved? On using chemical energy to support cardiac function," Circulation Research, vol. 95, no. 2, pp. 135-145, 2004.

[18] D. Von Lewinski, S. Bruns, S. Walther, H. Kögler, and B. Pieske, "Insulin causes $[\mathrm{Ca} 2+]$ idependent and $[\mathrm{Ca} 2+] \mathrm{i}$-independent positive inotropic effects in failing human myocardium," Circulation, vol. 111, no. 20, pp. 2588-2595, 2005.

[19] D. Von Lewinski, R. Gasser, P. P. Rainer et al., "Functional effects of glucose transporters in human ventricular myocardium," European Journal of Heart Failure, vol. 12, no. 2, pp. 106-113, 2010.

[20] D. von Lewinski, P. P. Rainer, R. Gasser et al., "Glucosetransporter-mediated positive inotropic effects in human myocardium of diabetic and nondiabetic patients," Metabolism: Clinical and Experimental, vol. 59, no. 7, pp. 1020-1028, 2010.

[21] H.-P. Hermann, B. Pieske, E. Schwarzmüller, J. Keul, H. Just, and G. Hasenfuss, "Haemodynamic effects of intracoronary pyruvate in patients with congestive heart failure: An open study," Lancet, vol. 353, no. 9161, pp. 1321-1323, 1999.

[22] G. Hasenfuss, L. S. Maier, H.-P. Hermann et al., "Influence of pyruvate on contractile performance and $\mathrm{Ca} 2+$ cycling in isolated failing human myocardium," Circulation, vol. 105, no. 2, pp. 194-199, 2002.

[23] A. M. From, C. L. Leibson, F. Bursi et al., "Diabetes in heart failure: prevalence and impact on outcome in the population," The American Journal of Medicine, vol. 119, no. 7, pp. 591-599, 2006.

[24] C. Iribarren, A. J. Karter, A. S. Go et al., "Glycemic control and heart failure among adult patients with diabetes," Circulation, vol. 103, no. 22, pp. 2668-2673, 2001.

[25] B. M. Scirica, E. Braunwald, I. Raz et al., "Heart failure, saxagliptin, and diabetes mellitus: observations from the SAVOR-TIMI 53 randomized trial," Circulation, vol. 132, no. 15, pp. e121-e122, 2015.

[26] G. Savarese, C. D’Amore, M. Federici et al., "Effects of Dipeptidyl Peptidase 4 Inhibitors and Sodium-Glucose Linked coTransporter-2 Inhibitors on cardiovascular events in patients with type 2 diabetes mellitus: a meta-analysis," International Journal of Cardiology, vol. 220, pp. 595-601, 2016. 
[27] J. Kongwatcharapong, P. Dilokthornsakul, S. Nathisuwan, A. Phrommintikul, and N. Chaiyakunapruk, "Effect of dipeptidyl peptidase-4 inhibitors on heart failure: A meta-analysis of randomized clinical trials," International Journal of Cardiology, vol. 211, pp. 88-95, 2016.

[28] M. Luconi, G. Cantini, A. Ceriello, and E. Mannucci, "Perspectives on cardiovascular effects of incretin-based drugs: From bedside to bench, return trip," International Journal of Cardiology, vol. 241, pp. 302-310, 2017.

[29] W. S. D. Silva Júnior, A. F. D. Godoy-Matos, and L. G. KraemerAguiar, "Dipeptidyl peptidase 4: a new link between diabetes mellitus and atherosclerosis?" BioMed Research International, vol. 2015, Article ID 816164, 10 pages, 2015.

[30] N.-H. Kim, T. Yu, and D. H. Lee, "The nonglycemic actions of dipeptidyl peptidase-4 inhibitors," BioMed Research International, vol. 2014, Article ID 368703, 10 pages, 2014.

[31] J. Zhong, A. Maiseyeu, S. N. Davis, and S. Rajagopalan, “DPP4 in cardiometabolic disease: recent insights from the laboratory and clinical trials of DPP4 inhibition," Circulation Research, vol. 116, no. 8, pp. 1491-1504, 2015.

[32] P. Balakumar and S. A. Dhanaraj, "Cardiovascular pleiotropic actions of DPP-4 inhibitors: A step at the cutting edge in understanding their additional therapeutic potentials," Cellular Signalling, vol. 25, no. 9, pp. 1799-1803, 2013.

[33] A. Solini, C. Rossi, E. Duranti, S. Taddei, A. Natali, and A. Virdis, "Saxagliptin prevents vascular remodeling and oxidative stress in $\mathrm{db} / \mathrm{db}$ mice. Role of endothelial nitric oxide synthase uncoupling and cyclooxygenase," Vascular Pharmacology, vol. 76, pp. 62-71, 2015.

[34] A. C. Keller, L. A. Knaub, M. W. Miller, N. Birdsey, D. J. Klemm, and J. E. B. Reusch, "Saxagliptin restores vascular mitochondrial exercise response in the Goto-Kakizaki rat," Journal of Cardiovascular Pharmacology, vol. 65, no. 2, pp. 137147, 2014.

[35] R. P. Mason, R. F. Jacob, R. Kubant, A. Ciszewski, J. J. Corbalan, and T. Malinski, "Dipeptidyl peptidase- 4 inhibition with saxagliptin enhanced nitric oxide release and reduced blood pressure and sICAM-1 levels in hypertensive rats," Journal of Cardiovascular Pharmacology, vol. 60, no. 5, pp. 467-473, 2012.

[36] K. A. Connelly, A. Advani, Y. Zhang et al., "Dipeptidyl peptidase-4 inhibition improves cardiac function in experimental myocardial infarction: role of stromal cell-derived factor-1 $\alpha$," Journal of Diabetes, vol. 8, no. 1, pp. 63-75, 2016.

[37] J. A. Hiemstra, D. I. Lee, K. Chakir et al., "Saxagliptin and tadalafil differentially alter cyclic guanosine monophosphate (cGMP) signaling and left ventricular function in aorticbanded mini-swine," Journal of the American Heart Association, vol. 5, no. 4, Article ID e003277, 2016.

[38] L. B. Katsnelson, T. Sulman, O. Solovyova, and V. S. Markhasin, "Role of myocardial viscoelasticity in disturbances of electrical and mechanical activity in calcium overloaded cardiomyocytes: mathematical modeling," Journal of Theoretical Biology, vol. 272, pp. 83-95, 2011.

[39] S.-T. Tang, H. Su, Q. Zhang et al., "Sitagliptin inhibits endothelin-1 expression in the aortic endothelium of rats with streptozotocin-induced diabetes by suppressing the nuclear factor-B/IB system through the activation of AMP-activated protein kinase," International Journal of Molecular Medicine, vol. 37, no. 6, pp. 1558-1566, 2016.
[40] K. A. Connelly, Y. Zhang, A. Advani et al., "DPP-4 inhibition attenuates cardiac dysfunction and adverse remodeling following myocardial infarction in rats with experimental diabetes," Cardiovascular Therapeutics, vol. 31, no. 5, pp. 259-267, 2013.

[41] T.-M. Lee, W.-T. Chen, and N.-C. Chang, "Sitagliptin decreases ventricular arrhythmias by attenuated glucose-dependent insulinotropic polypeptide (GIP)-dependent resistin signalling in infarcted rats," Bioscience Reports, vol. 36, no. 2, Article ID e00307, 2016.

[42] T.-M. Lee, W.-T. Chen, and N.-C. Chang, "Dipeptidyl peptidase4 inhibition attenuates arrhythmias via a protein kinase adependent pathway in infarcted hearts," Circulation Journal, vol. 79, no. 11, pp. 2461-2470, 2015.

[43] T.-I. Lee, Y.-H. Kao, Y.-C. Chen, J.-H. Huang, M.-I. Hsu, and Y.-J. Chen, "The dipeptidyl peptidase-4 inhibitor-sitagliptin modulates calcium dysregulation, inflammation, and PPARs in hypertensive cardiomyocytes," International Journal of Cardiology, vol. 168, no. 6, pp. 5390-5395, 2013.

[44] G. Esposito, D. Cappetta, R. Russo et al., "Sitagliptin reduces inflammation, fibrosis and preserves diastolic function in a rat model of heart failure with preserved ejection fraction," British Journal of Pharmacology, 2017.

[45] M. A. Alam, M. R. Hasan Chowdhury, P. Jain, M. A. T. Sagor, and H. M. Reza, "DPP-4 inhibitor sitagliptin prevents inflammation and oxidative stress of heart and kidney in two kidney and one clip (2K1C) rats," Diabetology and Metabolic Syndrome, vol. 7, no. 1, article no. 95, 2015.

[46] N. Hamdani, A.-S. Hervent, L. Vandekerckhove et al., "Left ventricular diastolic dysfunction and myocardial stiffness in diabetic mice is attenuated by inhibition of dipeptidyl peptidase 4," Cardiovascular Research, vol. 104, no. 3, pp. 423-431, 2014.

[47] M. Aoyama, H. Kawase, Y. K. Bando, A. Monji, and T. Murohara, "Dipeptidyl Peptidase 4 Inhibition Alleviates Shortage of Circulating Glucagon-Like Peptide-1 in Heart Failure and Mitigates Myocardial Remodeling and Apoptosis via the Exchange Protein Directly Activated by Cyclic AMP 1/RasRelated Protein 1 Axis," Circulation: Heart Failure, vol. 9, no. 1, 2016.

[48] K. Akita, K. Isoda, K. Shimada, and H. Daida, "Dipeptidylpeptidase-4 inhibitor, alogliptin, attenuates arterial inflammation and neointimal formation after injury in low-density lipoprotein (LDL) receptor-deficient mice," Journal of the American Heart Association, vol. 4, no. 3, Article ID e001469, 2015.

[49] S. Kato, K. Fukui, H. Kirigaya et al., "Inhibition of DPP-4 by alogliptin improves coronary flow reserve and left ventricular systolic function evaluated by phase contrast cine magnetic resonance imaging in patients with type 2 diabetes and coronary artery disease," International Journal of Cardiology, vol. 223, pp. 770-775, 2016.

[50] M. Yin, H. H. W. Silljé, M. Meissner, W. H. Van Gilst, and R. A. De Boer, "Early and late effects of the DPP-4 inhibitor vildagliptin in a rat model of post-myocardial infarction heart failure," Cardiovascular Diabetology, vol. 10, article no. 85, 2011.

[51] K. Chinda, J. Sanit, S. Chattipakorn, and N. Chattipakorn, "Dipeptidyl peptidase-4 inhibitor reduces infarct size and preserves cardiac function via mitochondrial protection in ischaemia-reperfusion rat heart," Diabetes and Vascular Disease Research, vol. 11, no. 2, pp. 75-83, 2014.

[52] T. Miyoshi, K. Nakamura, M. Yoshida et al., "Effect of vildagliptin, a dipeptidyl peptidase 4 inhibitor, on cardiac hypertrophy induced by chronic beta-adrenergic stimulation in rats," Cardiovascular Diabetology, vol. 13, no. 1, article 43, 2014. 
[53] M. Ishii, R. Shibata, K. Kondo et al., "Vildagliptin stimulates endothelial cell network formation and ischemia-induced revascularization via an endothelial nitric-oxide synthasedependent mechanism," Journal of Biological Chemistry, vol. 289, no. 39, pp. 27235-27245, 2014.

[54] N. Apaijai, H. Pintana, S. C. Chattipakorn, and N. Chattipakorn, "Effects of vildagliptin versus sitagliptin, on cardiac function, heart rate variability and mitochondrial function in obese insulin-resistant rats," British Journal of Pharmacology, vol. 169, no. 5, pp. 1048-1057, 2013.

[55] A. R. Aroor, J. R. Sowers, S. B. Bender et al., "Dipeptidylpeptidase inhibition is associated with improvement in blood pressure and diastolic function in insulin-resistant male zucker obese rats," Endocrinology, vol. 154, no. 7, pp. 2501-2513, 2013.

[56] V. Vellecco, E. Mitidieri, A. Gargiulo et al., "Vascular effects of linagliptin in non-obese diabetic mice are glucose-independent and involve positive modulation of the endothelial nitric oxide synthase (eNOS)/caveolin-1 (CAV-1) pathway," Diabetes, Obesity and Metabolism, vol. 18, no. 12, pp. 1236-1243, 2016.

[57] X.-W. Wang, F.-X. Zhang, F. Yang et al., "Effects of linagliptin and liraglutide on glucose-and angiotensin II-induced collagen formation and cytoskeleton degradation in cardiac fibroblasts in vitro," Acta Pharmacologica Sinica, vol. 37, no. 10, pp. 13491358, 2016.

[58] M. A. Pfeffer, B. Claggett, R. Diaz et al., "Lixisenatide in patients with type 2 diabetes and acute coronary syndrome," The New England Journal of Medicine, vol. 373, no. 23, pp. 2247-2257, 2015.

[59] S. P. Marso, G. H. Daniels, K. B. Frandsen et al., "Liraglutide and cardiovascular outcomes in type 2 diabetes," New England Journal of Medicine, vol. 375, no. 4, pp. 311-322, 2016.

[60] S. P. Marso, S. C. Bain, A. Consoli et al., "Semaglutide and cardiovascular outcomes in patients with type 2 diabetes," New England Journal of Medicine, vol. 375, no. 19, pp. 1834-1844, 2016.

[61] R. R. Holman, M. A. Bethel, J. George et al., "Rationale and design of the EXenatide Study of Cardiovascular Event Lowering (EXSCEL) trial," American Heart Journal, vol. 174, pp. 103$110,2016$.

[62] S. Mojsov, G. Heinrich, I. B. Wilson, M. Ravazzola, L. Orci, and J. F. Habener, "Preproglucagon gene expression in pancreas and intestine diversifies at the level of post-translational processing," Journal of Biological Chemistry, vol. 261, no. 25, pp. 11880-11889, 1986.

[63] T. J. Kieffer, C. H. S. McIntosh, and R. A. Pederson, "Degradation of glucose-dependent insulinotropic polypeptide and truncated glucagon-like peptide 1 in vitro and in vivo by dipeptidyl peptidase IV," Endocrinology, vol. 136, no. 8, pp. 3585-3596, 1995.

[64] V. Serre, W. Dolci, E. Schaerer et al., "Exendin-(9-39) is an inverse agonist of the murine glucagon-like peptide-1 receptor: Implications for basal intracellular cyclic adenosine $3^{\prime}, 5^{\prime}$-monophosphate levels and $\beta$-cell glucose competence," Endocrinology, vol. 139, no. 11, pp. 4448-4454, 1998.

[65] J. J. Meier, "GLP-1 receptor agonists for individualized treatment of type 2 diabetes mellitus," Nature Reviews Endocrinology, vol. 8, no. 12, pp. 728-742, 2012.

[66] D. J. Drucker and M. A. Nauck, "The incretin system: glucagonlike peptide-1 receptor agonists and dipeptidyl peptidase- 4 inhibitors in type 2 diabetes," The Lancet, vol. 368, no. 9548, pp. 1696-1705, 2006.
[67] L. A. Nikolaidis, S. Mankad, G. G. Sokos et al., "Effects of glucagon-like peptide-1 in patients with acute myocardial infarction and left ventricular dysfunction after successful reperfusion," Circulation, vol. 109, no. 8, pp. 962-965, 2004.

[68] J. Koska, E. A. Schwartz, M. P. Mullin, D. C. Schwenke, and P. D. Reaven, "Improvement of postprandial endothelial function after a single dose of exenatide in individuals with impaired glucose tolerance and recent-onset type 2 diabetes," Diabetes Care, vol. 33, no. 5, pp. 1028-1030, 2010.

[69] J. Lonborg, N. Vejlstrup, H. Kelbæk et al., "Exenatide reduces reperfusion injury in patients with ST-segment elevation myocardial infarction," European Heart Journal, vol. 33, no. 12, pp. 1491-1499, 2012.

[70] M. Tate, A. Chong, E. Robinson, B. D. Green, and D. J. Grieve, "Selective targeting of glucagon-like peptide-1 signalling as a novel therapeutic approach for cardiovascular disease in diabetes," British Journal of Pharmacology, vol. 172, no. 3, pp. 721-736, 2015.

[71] M. Kim, M. J. Platt, T. Shibasaki et al., "GLP-1 receptor activation and Epac2 link atrial natriuretic peptide secretion to control of blood pressure," Nature Medicine, vol. 19, no. 5, pp. 567-575, 2013.

[72] P. Richards, H. E. Parker, A. E. Adriaenssens et al., "Identification and characterization of GLP-1 receptor-expressing cells using a new transgenic mouse model," Diabetes, vol. 63, no. 4, pp. 1224-1233, 2014.

[73] P. Wohlfart, W. Linz, T. Hübschle et al., "Cardioprotective effects of lixisenatide in rat myocardial ischemia-reperfusion injury studies," Journal of Translational Medicine, vol. 11, no. 1, article no. 84, 2013.

[74] M. Wallner, E. Kolesnik, K. Ablasser et al., "Exenatide exerts a PKA-dependent positive inotropic effect in human atrial myocardium: GLP-1R mediated effects in human myocardium," Journal of Molecular and Cellular Cardiology, vol. 89, pp. 365375, 2015.

[75] J. L. Bos, "Epac proteins: multi-purpose cAMP targets," Trends in Biochemical Sciences, vol. 31, no. 12, pp. 680-686, 2006.

[76] O. Cazorla, A. Lucas, F. Poirier, A. Lacampagne, and F. Lezoualc'h, "The cAMP binding protein Epac regulates cardiac myofilament function," National Academy of Sciences of the United States of America, vol. 106, no. 33, pp. 14144-14149, 2009.

[77] N. J. Freedman, S. B. Liggett, D. E. Drachman, G. Pei, M. G. Caron, and R. J. Lefkowitz, "Phosphorylation and desensitization of the human $\beta 1$-adrenergic receptor: Involvement of $G$ protein-coupled receptor kinases and cAMP-dependent protein kinase," Journal of Biological Chemistry, vol. 270, no. 30, pp. 17953-17961, 1995.

[78] D. G. Tilley, A. D. Nguyen, and H. A. Rockman, “Troglitazone stimulates $\beta$-arrestin-dependent cardiomyocyte contractility via the angiotensin II type $1 \mathrm{~A}$ receptor," Biochemical and Biophysical Research Communications, vol. 396, no. 4, pp. 921926, 2010.

[79] D. G. Tilley, "G protein-dependent and G protein-independent signaling pathways and their impact on cardiac function," Circulation Research, vol. 109, no. 2, pp. 217-230, 2011.

[80] S. Mangmool, A. K. Shukla, and H. A. Rockman, " $\beta$-Arrestindependent activation of $\mathrm{Ca} 2+/$ calmodulin kinase II after $\beta 1$ adrenergic receptor stimulation," Journal of Cell Biology, vol. 189, no. 3, pp. 573-587, 2010.

[81] B. Zinman, C. Wanner, J. M. Lachin et al., "Empagliflozin, cardiovascular outcomes, and mortality in type 2 diabetes," The 
New England Journal of Medicine, vol. 373, no. 22, pp. 2117-2128, 2015.

[82] B. Neal, V. Perkovic, K. W. Mahaffey et al., "Canagliflozin and cardiovascular and renal events in type 2 diabetes," New England Journal of Medicine, 2017.

[83] N. Sattar, J. McLaren, S. L. Kristensen, D. Preiss, and J. J. McMurray, "SGLT2 Inhibition and cardiovascular events: why did EMPA-REG Outcomes surprise and what were the likely mechanisms?" Diabetologia, vol. 59, no. 7, pp. 1333-1339, 2016.

[84] E. Ferrannini, M. Mark, and E. Mayoux, "CV protection in the EMPA-REG OUTCOME trial: A thrifty substrate hypothesis," Diabetes Care, vol. 39, no. 7, pp. 1108-1114, 2016.

[85] J. Chen, S. Williams, S. Ho et al., "Quantitative PCR tissue expression profiling of the human SGLT2 gene and related family members," Diabetes Therapy, vol. 1, no. 2, pp. 57-92, 2010.

[86] S. A. Hawley, R. J. Ford, B. K. Smith et al., "The Na+/glucose cotransporter inhibitor canagliflozin activates AMPK by inhibiting mitochondrial function and increasing cellular AMP levels," Diabetes, vol. 65, no. 9, pp. 2784-2794, 2016.

[87] A. Baartscheer, C. A. Schumacher, R. C. I. Wüst et al., "Empagliflozin decreases myocardial cytoplasmic $\mathrm{Na}+$ through inhibition of the cardiac $\mathrm{Na}+/ \mathrm{H}+$ exchanger in rats and rabbits," Diabetologia, vol. 60, no. 3, pp. 568-573, 2017.

[88] J. Habibi, A. R. Aroor, J. R. Sowers et al., "Sodium glucose transporter 2 (SGLT2) inhibition with empagliflozin improves cardiac diastolic function in a female rodent model of diabetes," Cardiovascular Diabetology, vol. 16, no. 1, 2017.

[89] UK Prospective Diabetes Study (UKPDS) Group, "Effect of intensive blood-glucose control with metformin on complications in overweight patients with type 2 diabetes (UKPDS 34)," The Lancet, vol. 352, no. 9131, pp. 854-865, 1998.

[90] C. Lamanna, M. Monami, N. Marchionni, and E. Mannucci, "Effect of metformin on cardiovascular events and mortality: a meta-analysis of randomized clinical trials," Diabetes, Obesity \& Metabolism, vol. 13, no. 3, pp. 221-228, 2011.

[91] P. G. Steg and R. Roussel, "Randomized trials to evaluate cardiovascular safety of antihyperglycemic medications," Circulation, vol. 134, no. 8, pp. 571-573, 2016. 


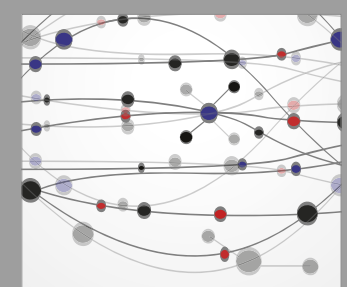

The Scientific World Journal
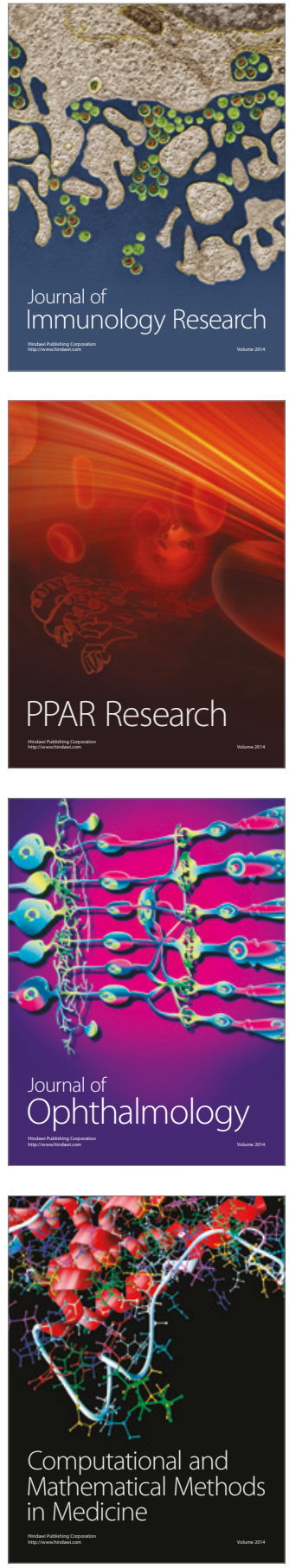

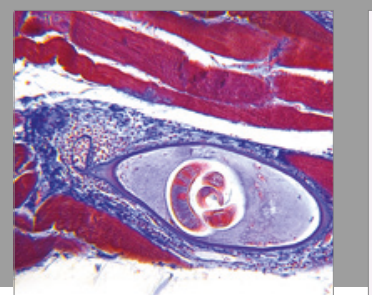

Gastroenterology Research and Practice
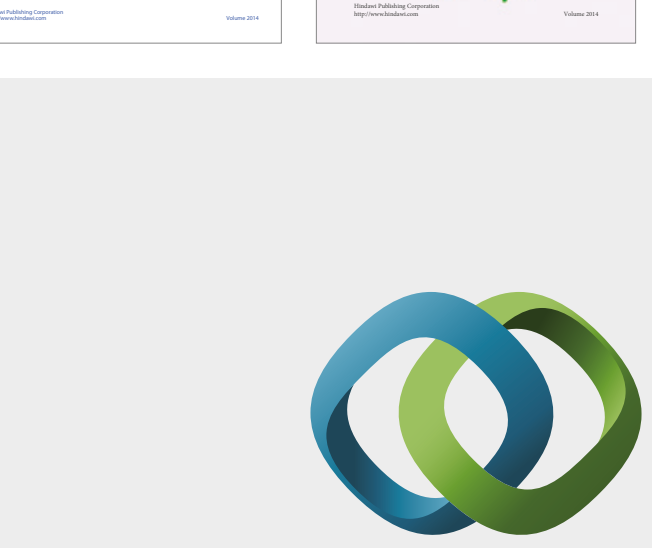

\section{Hindawi}

Submit your manuscripts at

https://www.hindawi.com
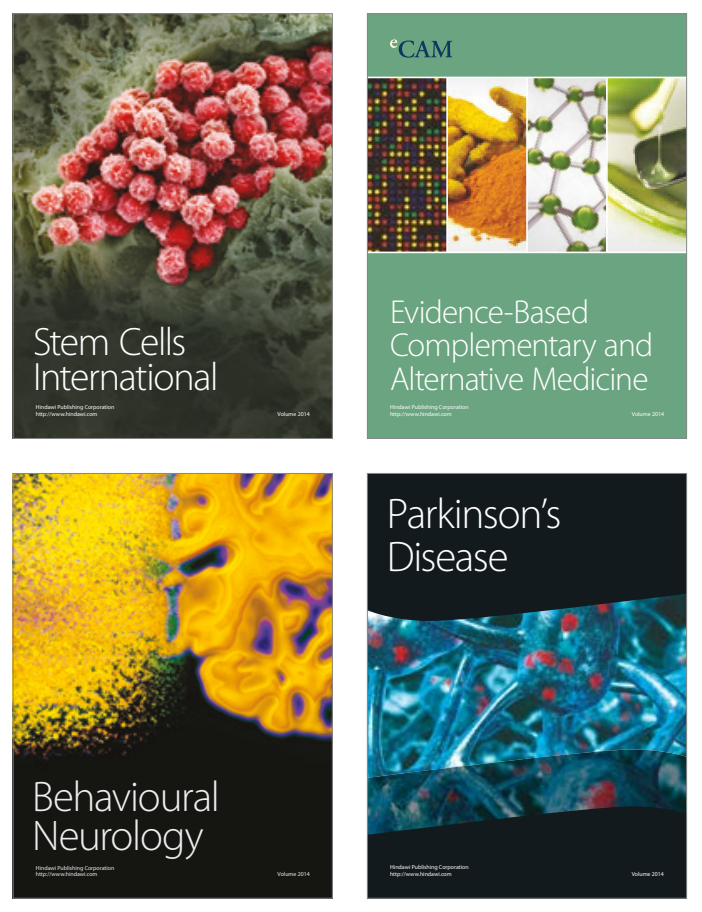
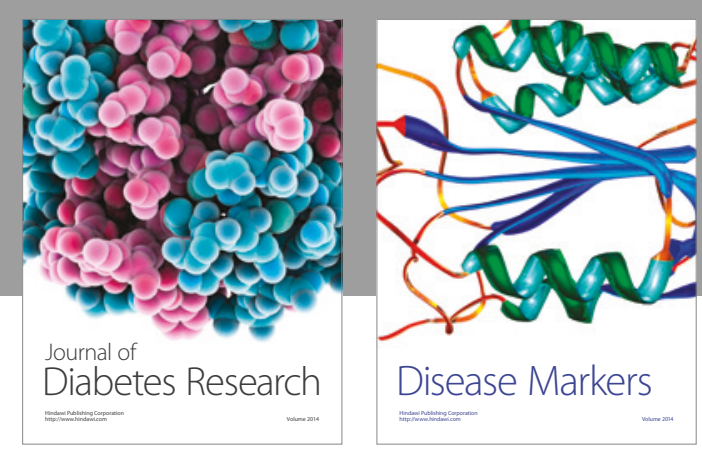

Disease Markers
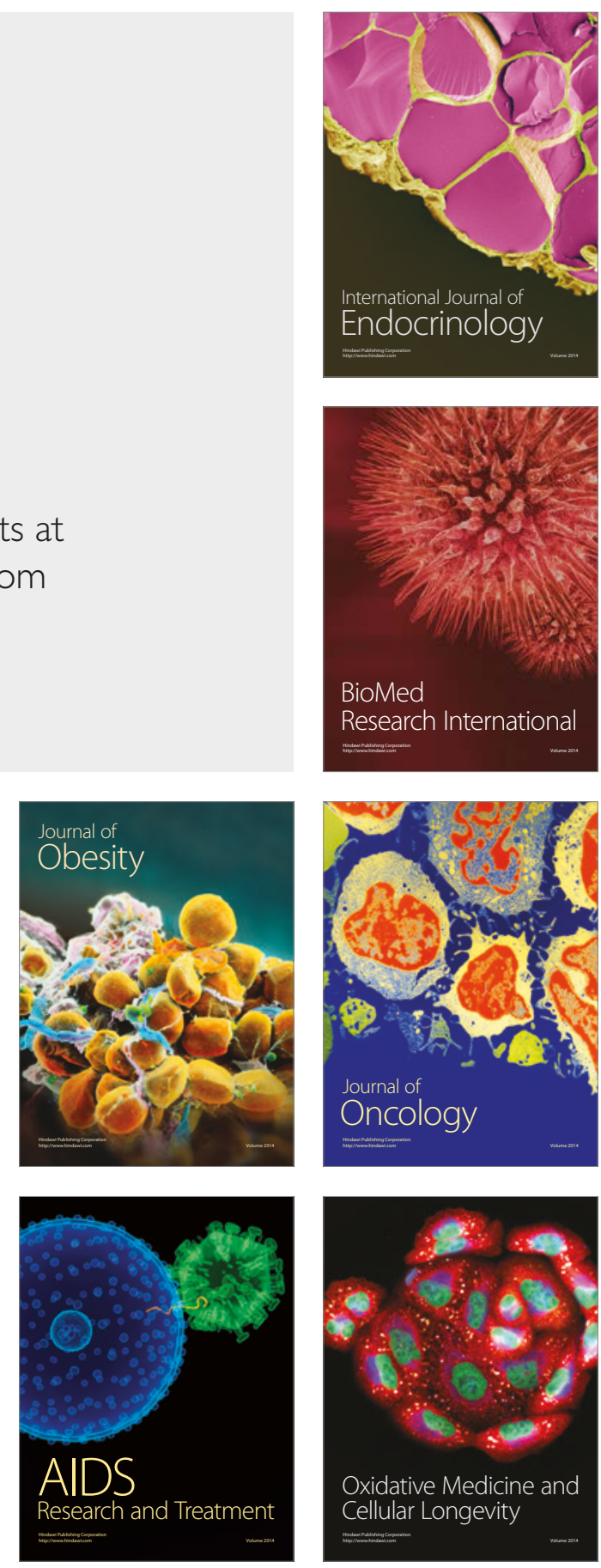